\title{
Atrioventricular Pacemaker. Incidence and Causes of Reprogramming in Long-term Follow-Up
}

\author{
Martino Martinelli Fo, Sylton de A rruda Melo, Silvana A. D. N ishióka, A nísio A. A. Pedrosa, Sérgio \\ Freitas de Siqueira, Roberto Costa, Eduardo Sosa
}

São Paulo, SP - Brazil

\begin{abstract}
Objective - To assess the incidence of problems requiring reprogramming of atrioventricular pacemakers in a long-termfollow-up, and also the causes for this procedure.

Methods - During the period from May '98 to December '99, 657 patients were retrospectively studied, An actuarial curve for the event reprogramming of the stimulation mode was drawn.
\end{abstract}

Results - The follow-up period ranged from 12 to 178 months (mean $=81$ months). Eighty-two $(12.4 \%)$ patients underwent reprogramming of the stimulation mode as follows: $63(9.5 \%)$ changed to $V V I,(R / C) ; 10(1.5 \%)$ changed to DVI,C; $6(0.9 \%)$ changed to VDD,C; and $3(0.5 \%)$ changed to DOO. The causes for the reprogramming were as follows: arrhythmia conducted by the pacemaker in 39 (37.6\%) patients; loss of atrial sensitivity or capture, or both, in 39 (38.6\%) patients; and microfracture of atrial electrode in $5(4.9 \%)$ patients. The stimulation mode reprogramming free probability after 15 years was $58 \%$.

Conclusion - In a long-term follow-up, the atrioventricular pacemaker provided a low incidence of complications, a high probability of permanence in the $D D D, C$ mode, and the most common cause of reprogramming was arrhythmia conducted by the pacemaker.

Key words atrioventricular pacemaker, reprogramming, DDD,Cmode
Instituto do Coração do Hospital das Clínicas - FMUSP

Mailing address: Martino Martinelli $F^{o}-$ InCor - Av. Dr. Enéas C. Aguiar $44-$ 05403-000 - São Paulo, SP, Brazil

English version by Stela Maris C. Gandour
Atrioventricular stimulation, introduced by Nathan et al ${ }^{1}$ in 1963 with the ventricular pacing synchronous with the $\mathrm{P}$ wave, has been greatly improved since the 80 's with the appearance of specific algorithmic functions, lithium batteries, and atrial electrodes of active fixation ${ }^{2}$.

Controversies in regard to the cost-benefit ratio of the ventricular (VVI,C) or the atrioventricular (DDD,C) pacemakers persist ${ }^{3-6}$. The major advantages related to dual chamber pacemakers are atrioventricular resynchronization in patients with atrioventricular block, and the ability to use the sinus node as a sensor, causing physiological variation in heart rate. In addition, in cases of sick sinus syndrome, atrioventricular stimulation prevents the consequences of the atrioventricular node disease progression, as well as the pacemaker syndrome ${ }^{7}$. Among the disadvantages of dual chamber pacing, we can cite its higher cost and technical difficulties for implantation and follow-up ${ }^{8}$.

The most commonly reported complications of the atrioventricular system are loss of atrial sensitivity, atrial arrhythmias, and alterations in the stimulation threshold ${ }^{9,10}$. However, the relation between these findings and the therapeutical procedures has not been established.

The objective of this study was to assess the incidence of complications requiring reprogramming of the atrioventricular stimulation systems in a long-term follow-up, and also the major causes for this procedure.

\section{Methods}

At the pacing clinic of the heart institute, 4,250 pacemaker patients are regularly followed up, 2,820 of whom have atrioventricular stimulation systems. Among these, we selected 657 patients, who, during the period from May ' 98 to December' 99 , came to our institution searching for regular (clinical and electronic) pacing assessment. All of them had been followed up for at least one year and were retrospectively assessed (medical record and database), with special attention to system reprogramming recordings and the causes for this procedure. These data underwent statistical 
analysis, and a curve of the accumulated probability of permanence in the DDD,C mode was drawn (KaplanMeier).

In the study, 355 patients were males and 302 were females. Their ages ranged from 7 to 92 years (mean $=58$ years and 2 months). The most common indication for pacemaker implantation was total atrioventricular block, observed in 359 patients. Other indications included the following: type II or advanced second-degree atrioventricular block in 138 patients, sick sinus syndrome in 115 patients, hypersensitivity of the carotid sinus in 34 patients, long QT syndrome in 8 patients, and others in 3 patients (Table I). Chagas' was the most common underlying heart disease present in 227 patients, followed by ischemic heart disease in 196 patients, hypertension in 92 patients, valvar heart disease in 71 patients, congenital heart disease in 28 patients, and other causes in 43 patients (Table II).

\section{Results}

The mean follow-up duration was 81 months, ranging from 12 to 178 months. At the end of the study, $101(15.3 \%)$ patients had undergone system reprogramming, $82(12.4 \%)$ of them for changes in the stimulation mode, as follows: 63 (9.5\%) changed to VVI,(R/C); $10(1.5 \%)$ changed to DVI,C; 6 $(0.9 \%)$ changed to VDD,C; and $3(0.5 \%)$ changed to DOO. The findings regarding the other types of reprogramming are shown in table III.

The causes for changing the stimulation mode were as follows: arrhythmia conducted by the pacemaker in 38 (37.6\%) patients; loss of atrial sensitivity or capture or both in $39(38.6 \%)$ patients; microfracture of the atrial electrode in $5(4.9 \%)$ patients; an increase in ventricular threshold in 8 (7.9\%) patients; pectoral muscle stimulation in 6 (5.9) patients; and alteration in ventricular sensitivity in $5(4.9 \%)$ patients (Table IV).

\begin{tabular}{|lll|}
\hline \multicolumn{2}{|c|}{ Table I - Indications for the implantation of a definitive pacemaker } \\
\hline \multicolumn{1}{|c|}{ Indication } & $\mathrm{N}$ & $\%$ \\
\hline Total atrioventricular block & 359 & 54.6 \\
Type II second degree atrioventricular block & 138 & 21.0 \\
Sick sinus syndrome & 115 & 17.5 \\
Hypersensitivity of the carotid sinus & 34 & 5.2 \\
Long QT syndrome & 8 & 1.2 \\
Others & 3 & 0.5 \\
\hline
\end{tabular}

\begin{tabular}{|lcc|}
\hline \multicolumn{2}{|c|}{ Table II - Prevalence of heart diseases } \\
\hline Type & N & $\%$ \\
\hline Chagas' Heart Disease & 227 & 34.6 \\
Ischemic Heart Disease & 196 & 29.8 \\
Hypertension & 92 & 14.0 \\
Valvar Heart Disease & 71 & 10.8 \\
Congenital Heart Disease & 28 & 4.3 \\
Others & 43 & 6.5 \\
\hline
\end{tabular}

\begin{tabular}{|lcc|}
\hline \multicolumn{3}{|c|}{ Table III - System reprogramming } \\
\hline Type & $\mathrm{n}$ & $\%$ \\
\hline Stimulation mode & 82 & 81.1 \\
Energy, atrial sensitivity or polarity, or both & 9 & 8.9 \\
Ventricular stimulation energy & 5 & 4.9 \\
Ventricular sensitivity & 5 & 4.9 \\
\hline
\end{tabular}

Forty-three patients underwent temporary reprogramming of the stimulation mode, 5 patients due to microfracture of the electrode (DOO mode) while awaiting surgical treatment and 38 patients due to arrhythmias conducted by the pacemaker. These latter underwent reprogramming for the VVI,C mode to undergo chemical or electrical cardioversion, and 15 of them did not return to the DDD,C mode because definitive reversion of the atrial rhythm to sinus rhythm was not possible.

The stimulation system reprogramming did not allow definitive correction in 13 patients who underwent surgical treatment. In regard to atrial leads, 3 changes and 5 repositionings were performed, and in regard to ventricular leads, 2 changes and 3 repositionings were performed.

Analysis of the event-free curve showed probability of reprogramming at 5,10 , and 15 years of $10 \%, 18 \%$, and $42 \%$, respectively. During follow-up, 12 deaths occurred (fig. 1).

\begin{tabular}{|lcc|}
\hline \multicolumn{2}{|c|}{ Table IV - Causes of system reprogramming } \\
\hline \multicolumn{1}{|c|}{ Causes } & $\mathrm{n}$ & $\%$ \\
\hline Arrhythmia conducted by the pacemaker & 38 & 37.6 \\
Loss of atrial capture & 21 & 20.8 \\
Atrial undersensing & 18 & 17.8 \\
Microfracture of atrial electrode & 5 & 4.9 \\
Increase in the ventricular threshold & 8 & 7.9 \\
Pectoral muscle stimulation & 6 & 5.9 \\
Ventricular undersensing & 5 & 4.9 \\
\hline
\end{tabular}

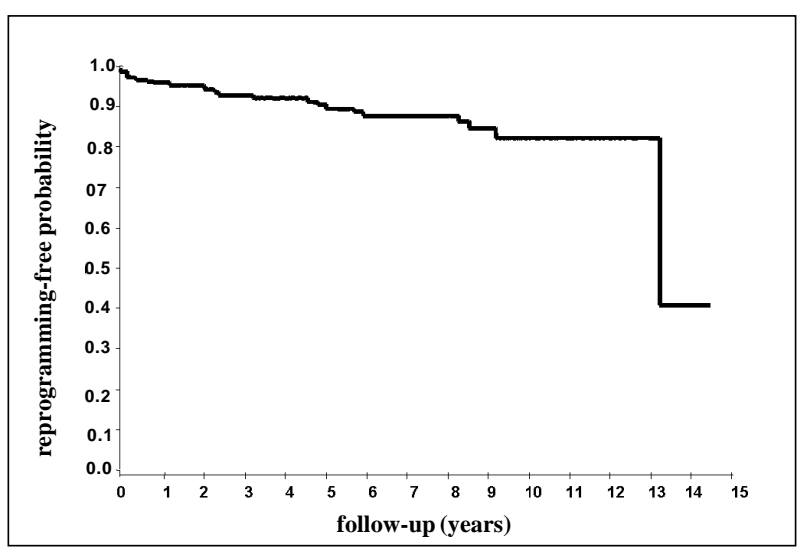

Fig. 1 - Actuarial curve of events (Kaplan-Meier) depicting the reprogramming-free probability of the stimulation mode throughout follow-up. Note that at 5 years, the reprogramming-free probability was $90 \%$, and at 15 years it was $58 \%$. 


\section{Discussion}

The extraordinary technological advance involving devices used for artificial cardiac stimulation throughout a relatively short period of time, from its introduction to the current days, has been marked by very well defined developmental phases. Starting with primary systems comprising one chamber with fixed frequency and no sensitivity, pacemakers with a progressive functional capacity have been manufactured until reaching the system characterized as physiological: atrioventricular, multifunctional, with frequency variation guided by the chronotropic response of the normal sinus node ${ }^{2,11-13}$.

Despite these favorable technological characteristics, the clinical consequences of this therapy have not yet been assessed enough to allow definitive conclusions. In addition, the complication rates attributed to the most sophisticated systems have been considered high and of difficult management, particularly for the nonspecialized physician ${ }^{3-6}$.

Several studies have shown the clinical and hemodynamic advantages of the DDD,C mode stimulation as compared with the VVI,C mode, both for total atrioventricular block and sick sinus syndrome ${ }^{4,6,10,14,15}$. The VVI,C pacemaker has been associated with a lower physical capacity $^{4,15}$, a worse quality of life ${ }^{6,10,15}$, an enlargement of the cardiac silhouette ${ }^{14}$, the pacemaker syndrome ${ }^{16}$, and a higher mortality rate ${ }^{17}$.

Other studies have shown no significant difference in regard to mortality ${ }^{3}$ or functional capacity ${ }^{5}$. Atrioventricular resynchronization, considered responsible for improving physical capacity by some authors ${ }^{4,14}$, was challenged by Chu-Pak Lau et al ${ }^{18}$, who attributed this increment basically to variations in heart rate.

Mueller et al ${ }^{19}$ have recently challenged the highest indices of complication attributed to DDD,C pacemakers by showing results similar to those of the ventricular systems (VVI,C).

In our study, rates related to reprogramming of the stimulation mode were low (12.4\%). Gross et al ${ }^{10}$, studying 486 patients in DDD,C mode, observed definitive change rates in $18 \%$ of the patients and established a relation between these findings and the presence of atrial fibrillation. We attribute these differences with our findings to criteria established by the authors in the therapeutical approach of atrial arrhythmia, more conservative in the indication of electrical cardioversion. Our protocol for this clinical situation is based on reestablishing sinus rhythm in regard to hemodynamic improvement, and also on the prophylaxis of thromboembolism. Electrical cardioversion is not contraindicated and should not be minimized in its indication, because as long as it is performed in special technical conditions and under the protection of the pacemaker generator, it is perfectly feasible. On the other hand, Markewitz et $\mathrm{al}^{20}$, without considering the causes for mode reprogramming in atrioventricular stimulation systems, observed very low indices (5\%). In this study, 345 patients enroled during 5 years were followed-up for 6 years, the main objective being the maintenance of atrioventricular synchronism, independent of the mode (DDD, DDI, DVI, VDD).

In regard to the causes for reprogramming the system, our study has shown that arrhythmia conducted by the pacemaker was the most common finding. In this category, we include all arrhythmias generated in the atrial chamber, such as fibrillation, tachycardia, atrial flutters, and others that trigger ventricular stimulation in an inadequate frequency and rhythm. In these cases, our general approach was temporary reprogramming to the VVI,C mode, immediate chemical or electrical cardioversion under the careful technique of protection and, depending on the result, the return to the DDD,C or DVI,C modes. In our study, this protocol was applied to 38 patients, and in 23 patients we obtained definitive success. Hummel et $\mathrm{al}^{21}$ and ChamberlainWebber et al ${ }^{9}$ in a smaller series of patients observed similar rates of complication, but they did not report the indices of therapeutical success. In our study, 5 patients did not return to the condition of atrioventricular stimulation, remaining in VVI,C mode, because of failure in the attempt of cardioversion to sinus rhythm. Several authors ${ }^{9,21-23}$ have correlated this with the evolution of the underlying heart disease, which is usually associated with aggravation of the congestive heart failure functional class. Detollenaere et $\mathrm{al}^{22}$ have shown that the incidence of these arrhythmias (12\%) does not correlate with mortality. Ibrahim et al ${ }^{23}$ have observed that in the presence of sick sinus syndrome, the risk of pacemaker patients for developing atrial fibrillation is 1.5 times higher than that of those with disorders in atrioventricular conduction.

In our study, dysfunctions related to the presence of the atrial pacemaker lead, such as loss of atrial sensitivity in 21 patients, and capture in 18 patients, had indices of complication close to the mean reported by Chamberlain-Webber et $\mathrm{al}^{9}$ and Gross et $\mathrm{al}^{10}$. These dysfunctions also seem to be associated with the anatomic and functional conditions of the atrial chamber. On the other hand, microfracture of the electrode, which is an entity characterized by disruption in the lead causing noises and inhibition of the stimulation, is exclusively inherent in a technical defect in the system. No reference to this complication exists in the literature, and our study has shown that in these cases temporary programming of the DOO is safe and adequately protects against pacemaker inhibition caused by a defect while awaiting surgery (change of the pacemaker lead). In fact, the 5 patients with this dysfunction revealed no symptoms of low cerebral blood flow, including the 2 patients who awaited surgery for a long time.

The incidence of changes in ventricular stimulation and sensitivity threshold, as well as pectoral muscle stimulation, did not differ from the classical rates shown by studies performed in ventricular systems.

Our percentage of surgery, including the cases refractory to reprogramming, was much lower than that reported by Gross et al $^{10}$ and Ibrahim et $\mathrm{al}^{23}$, whose values were 4 and $9.6 \%$, respectively. 
The reprogramming-free probability throughout the follow-up was assessed only in regard to the stimulation mode, because this was the most common therapeutical procedure performed (82/101). Through the actuarial curve of this event, we could document the rates of permanence in DDD, $C$ mode in regard to implantation time, the major aim of indicating a dual chamber system. The values found exceeded our expectation, as follows: by 5 years, only $10 \%$ of the patients did not remain in the initially programmed stimulation mode, and by 15 years, $58 \%$ of those who started the study remained in the DDD,C mode. These findings were not reported in the literature, which usually reports curves of events in patients with atrioventricular stimulation systems considering only mortality.

In conclusion, our study has shown that atrioventricular stimulation provides a low incidence of complications requiring reprogramming and a high probability of permanence in the initial stimulation mode (DDD,C) in a long-term follow-up. The causes of reprogramming were varied, the most common being arrhythmia conducted by the pacemaker.

\section{References}

1. Nathan DA, Center S, Wu CY, Keller W. An implantable synchronous pacemaker for the long-term correction of complete heart block. Circulation 1963;27: 682 .

2. Bronzino JD. Cardiac Pacing - Historical Highlights. The Biomedical Engineering Hand Book. Connecticut: Ed CRC Press, 1995; 87-95.

3. Lamas GA, Orav J, Stambler BS, et al. Quality of life and clinical outcomes in elderly patients treated with ventricular pacing as compared with dual-chamber pacing. N Eng J Med 1998; 338: 1097-104.

4. RedikerDE, Eagle KA, Homma S, Gillam LD, Harthorne JW. Clinical and Hemodynamic Comparasion of VVI Versus DDD Pacing in Patients with DDD Pacemakers. Am J Cardiol 1988; 61: 323-9.

5. Bubien RS, Kay GN. A Randomized comparation of quality of life and exercise capacity with DDD and VVIR pacing modes. PACE 1990; 13: 524.

6. Lukl J, Doupal V, Heinc P. Quality-of-life during DDD and dual sensor VVIR pacing. PACE 1994; 17: 1844-8.

7. McComb JM, Gribbin GM. Effect of pacing mode on morbidity and mortality: updating of clinical pacing trials. Am J Cardiol 1999; 11: 211D-213D.

8. Sutton R, Bourgeois I. Cost benefit analysis of single and dual chamber pacing for sick sinus syndrome and atrioventricular block. An economic sensitivity analysis of the literature. Eur Heart J 1996; 17: 574-82

9. Chamberlain-Webber R, Ingram PA, Briers L, Sutton R. Reasons for reprogramming dual chamber pacemakers to VVI mode: A retrospective review using a computer database. PACE 1994; 17: 1730-6.

10. Gross JN, Moser S, BenedekZM, Andrews C, Furman S. DDD pacing mode survival in patients with a dual-chamber pacemaker. J Am Coll Cardiol 1992; 19: 1536-41.

11. Camilli L. Results, Problems and Perspectives with an regulating pacemaker. PACE 1983; 6: 488.
12. Williams DO, Davidson PH. Long term treatment of refractory supraventricular tachycardia by patient controlled inductive atrial pacing. Br Heart J 1974; 36: 336

13. Wirtzfeld A. Central venous oxygem saturation for the control of automatic rate response pacing. PACE 1982; 5: 829.

14. Kruse I, Arnman K, Conradson T-B, Rydén L. A comparasion of the acute and long-term hemodynamic effects of ventricular inhibited and atrial synchronous ventricular inhibited pacing. Circulation 1982; 65: 846-55.

15. Frielingsdorf J, Gerber AE, Hess OM. Importance of maintained atrio-ventricular synchrony in patients with pacemakers. Eur Heart J 1994; 15: 1431-40.

16. Heldman D, Mulvihill D, Nguyen $\mathrm{H}$, et al. True incidence of pacemaker syndrome. PACE 1990; 13: 1742-50.

17. Hesselson $\mathrm{AB}$, Parsonnet $\mathrm{V}$, Bernstein $\mathrm{AD}$, Bonavita GJ. Deleterious effects of longterm single-chamber ventricular pacing in patients with sick sinus syndrome: The hidden benefits of dual-chamber pacing. J Am Coll Cardiol 1992; 19: 1542-9.

18. Lau C-P, Rushby J, Leigh-Jones M, et al. Symptomatology and quality of life in patients with rate-responsive pacemakers: A double-blind, randomized, crossover study. Clin Cardiol 1989; 12: 505-12.

19. Mueller X, Sadeghi H, Kappenberger. Complications after single versus dual chamber pacemaker implantations. PACE 1990; 13: 711-4.

20. Markewitz A, Hemmer W, Weinhold C. Complications in dual chamber pacing: A six-year experience. PACE 1986; 9: 1014-8.

21. Hummel J, Fazio G, Lawrence J, Midei M, Walford G, Briker J. The natural history of dual chamber system. PACE 1991; 14: 621

22. Detollenaere M, Wassenhove Van E, Jordaens. Atrial arrhythmias in dual chamber pacing and their influence on long-term mortality. PACE 1992; 15: 1846-50.

23. Ibrahim B, Sanderson JE, Wright B, Palmer R. Dual chamber pacing: How many patients remain in DDD mode over the long term? Br Heart J 1995; 74: 76-9. 